\title{
Prevalência de Hipovitaminose A em Crianças da Periferia do Município de Campinas, São Paulo, Brasil'
}

\author{
Prevalence of Hypovitaminosis A in Children of Peripheral Districts of \\ Campinas São Paulo, Brazil
}

\author{
Cecília Maria R. Gonçalves-Carvalho ; Jaime Amaya-Farfan³ ; \\ Berenice C. Wilke ${ }^{3} \&$ Roland Vencovsky ${ }^{4}$
}

GONÇALVES-CARVALHO, C. M. R; AMAYA-FARFAN, J.; WILKE, B. C. \& VENCOVSKY, R Prevalence of Hypovitaminosis A in Children of Peripheral Districts of Campinas São Paulo, Brazil. Cad. Saúde Públ., Rio de Janeiro, 11 (1): 85-96, Jan/Mar, 1995.

The prevalence of hypovitaminosis A among children of the peripheral districts of the city of Campinas, São Paulo, Brazil, was estimated by determining serum retinol levels by High Performance Liquid Chromatography (HPLC) in a sample of 131 children aged between three and ten years, between April 1991 and February 1992. A prevalence of $17.6 \%$ and retinol concentrations in the range of 0.35 to $0.70 \mu \mathrm{mol} / \mathrm{L}$ were found $(C I=11.1-24.1 ; 95 \%)$, indicating the existence of public health risk Ophthalmological examinations, however, failed to detect any cases of xerophthalmy. Additional characterization of the sample was obtained from 341 children. The per capita incorre of the average household was surprisingly high for low-income areas. According to FAO-WHO standards, food consumption was adequate only for protein (133.96\%). Adequacy levels were low for energy (87.76\%) and particularly for vitamin A (66.13\%) and iron (42.14\%). Height for-age and weight-for-height anthropometric indices revealed that many children were located below -1 standard deviation.

Key words: Vitamin A; Hypovitaminosis A; Retinol; Food Consumption; Anthropometry

\section{INTRODUÇÃO}

A hipovitaminose A é problema nutricional dos mais graves e que acomete, principalmente, crianças pobres. Estima-se que cerca de 25 a 50

\footnotetext{
${ }^{\prime}$ Os autores desejam reconhecer o apoio da Fundação de Amparo a Pesquisa de São Paulo, ao Conselho Nacional de Desenvolvimento Científico e Tecnológico e a cooperação da Secretaria Municipal da Saúde (Convênio Unicamp/PMC, LM no 6463 de 08/05/91).

${ }^{2}$ Departamento de Nutrição, Centro de Ciências da Saúde, Universidade Federal do Piauí, Campus Universitário Iningá, Teresina, PI, 64049-550, Brasil.

${ }^{3}$ Departamento de Planejamento Alimentar e Nutrição, Faculdade de Engenharia de Alimentos, Universidade Estadual de Campinas, Caixa Postal 6121, Campinas, SP, 13081-970, Brasil.

${ }^{4}$ Departamento de Genética, Escola Superior de Agricultura Luiz de Queiroz. Avenida Pádua Dias, 11, Caixa Postal 9, Piracicaba, SP, 13418-260, Brasil.
}

milhões de crianças no mundo, com ou sem sinais de xeroftalmia, desenvolvam deficiência de vitamina A (Bloem et al., 1990) e, a cada ano, entre 100.000 e 250.000 fiquem cegas permanentemente (Sommer, 1989a).

Além das alterações oculares, retardo no crescimento (Sommer, 1989b), aumento da susceptibilidade às infecções (Sommer et al., 1987), aumento do risco de doenças respiratórias e diarréias (Sole et al., 1987; Sommer et al., 1987) são outras graves conseqüências dessa deficiência. Na maioria das vezes, a deficiência de vitamina A aparece associada à desnutrição protéico-energética (Carlier et al., 1991), sendo que as formas avançadas da hipovitaminose A evoluem espontaneamente para o óbito.

Mesmo nas suas formas mais leves, a deficiência de vitamina A aparece como fator importante na determinação da morbidade e da mortalidade na infância (Sommer et al., 1983). 
É necessário, portanto, que os casos marginais dessa deficiência sejam diagnosticados tão cedo quanto possível, especialmente em populações de maior risco, para que medidas de prevenção e controle sejam implementadas.

Apesar de suas graves conseqüências, no Brasil pouco se conhece sobre a profundidade e extensão da hipovitaminose A. Muitos casos de deficiência subclínica com possibilidades de desenvolvimento de lesões oculares devem existir, uma vez que o consumo inadequado de vitamina A e carotenóides tem sido apontado como problema de saúde pública em várias regiões do país (Silva, 1959; ICNND, 1965; Shrimpton, 1984). No Estado de São Paulo, os inquéritos nutricionais, clínicos e bioquímicos revelam existir deficiência de vitamina A (Mazilli \& Gandra, 1981; Wilson et al., 1981; Roncada et al., 1984; Wilson \& Roncada, 1985; Favaro et al., 1986).

Considerando que no Município de Campinas, São Paulo, não existem estudos populacionais sobre a deficiência de vitamina $\mathrm{A}$, decidiu-se determinar a prevalência de hipovitaminose A em crianças residentes em favelas desse município, utilizando-se as dosagens de retinol no soro por cromatografia liquida de alta eficiência (Clae).

\section{MATERIAL E MÉTODOS}

\section{Local e Época da Pesquisa}

O estudo foi conduzido na periferia do município de Campinas no período de março de 1991 a fevereiro de 1992. Campinas é a segunda cidade do estado de São Paulo, com área de $801 \mathrm{Km}^{2}$, e população estimada de 846.238 habitantes para o ano de 1991. A população favelada compreende cerca de 67.474 pessoas, distribuídas em 14.248 barracos (Campinas, 1993).

\section{Critérios de Seleção}

Só foram incluídas no estudo crianças que tivessem consentimento prévio assinado pelos pais ou responsáveis. Foram excluídas crianças com histórico de febre ou infecções nos cinco dias anteriores à data de realização da pesquisa.

\section{Cálculo e Processo de Amostragem}

Em maio de 1991, havia 103 favelas distribuídas nas 13 Áreas Regionais (AR's) do Munícipio de Campinas. Dezoito favelas foram sorteadas, em função da capacidade de operacionalização da pesquisa (tempo e recursos disponíveis), e distribuídas em quatro grupos. Para diminuir a variabilidade do estimador, tentou-se fazer associação homogênea entre as diversas AR's, levando em consideração as particularidades de cada local, como foi a proximidade ou não do Ceasa, de modo a eliminar a influência da maior oferta de alimentos fontes de vitamina A. A divisão em grupos foi assim caracterizada:

- Grupo 1 (AR 5, 6, 7, 11, 12, 13, duas sub prefeituras e dois subdistritos), região mais distante do Centro da cidade, separada pela via Anhanguera;

- Grupo 2 (Ar 2 e 3), região mais próxima ao Shopping Center Iguatemi;

- Grupo 3 (AR 4), região mais próxima ao Ceasa;

- Grupo 4 (AR9 e 10), região ao leste da via Anhanguera.

O sorteio das favelas dentro de cada grupo foi feito utilizando-se a tabela de números aleatórios de Cochran (1977). Da mesma forma, a seqüência de participação de cada favela foi também aleatória.

Foi realizado um estudo-piloto em 100 crianças avaliadas por citologia de impressão da conjuntiva ocular (Cico), em que $2 \%$ apresentavam deficiência de vitamina $\mathrm{A}$ ou $98 \%$ não apresentavam a característica. Para esse cálculo, considerou-se o nível de significância de $5 \%$ e estimou-se a diferença de $2 \%$ entre a proporção amostral e a populacional (Armitage \& Berry, 1987).

Como não se conhecia o número de residências por favela, determinou-se arbitrariamente que o total de domícilios visitados corresponderia a $10 \%$. Em cada favela sorteada manteve-se a proporção de um para cada 10 domicílios, sendo selecionadas 229 famílias para o total de 350 crianças. 
Das 350 crianças selecionadas foi retirada uma subamostra $(\mathrm{n}=138)$, para as dosagens séricas de retinol, em função da aceitação das crianças e famílias e do custo financeiro para a realização desses exames.

\section{Características da Amostra}

Para caracterizar a amostra, investigaram-se as seguintes variáveis: renda familiar, número de pessoas da família, instrução dos pais, antropometria, inquérito alimentar e exame clínico ocular. Em cada domícilio visitado foi feito esclarecimento às famílias sobre objetivos e finalidades da pesquisa. As crianças que apresentavam qualquer suspeita de doença eram encaminhadas aos serviços médicos do Centro de Saúde de cada região.

A renda familiar foi obtida em salário mínimo da época de realização da pesquisa, e a renda per capita, a partir da divisão da renda total pelo número de indíviduos da família. Foram considerados para o cálculo do número de pessoas da família todos aqueles que viviam no mesmo domícilio e se beneficiavam da renda familiar.

O grau de instrução dos pais foi assim classificado: analfabeto (com menos de um ano de estudo); primário incompleto (um a três anos de estudo); primário completo (quatro anos de estudo concluído); e ginasial (cinco a oito anos de estudo).

\section{Métodos}

\section{Antropometria}

A idade, pela certidão de nascimento, e as medidas de peso e altura foram relacionadas com as da população-referência do National Center for Health Statistics (NCHS) (OMS, 1983) por meio de escores de desvio padrão (z score). $\mathrm{O}$ cálculo dos escores z foram feitos por softwares desenvolvidos pelo Center for Disease Control, em Atlanta, GA, EUA (Anthropometric software package), descritos por Jordan (1987).

\section{Inquérito Alimentar}

Em entrevista com a mãe ou responsável pela criança, foram colhidas informações sobre consumo alimentar, por meio da aplicação de inquérito recordatório de 24 horas. O cálculo dos nutrientes para cada criança foi obtido usando-se o Programa Computadorizado de Cálculo de Dietas do Centro de Informática em Saúde, da Escola Paulista de Medicina (Anção et al., s/d). Para a análise de adequação alimentar, relacionou-se o requerimento correspondente (FAO, 1985, 1988) com as quantidades de ingesta declaradas.

Os valores de vitamina A para alface, salsinha, rúcula, agrião, couve, almeirão, chicória, abóbora, tomate, manga, mamão, pimentão, brócoli e cenoura foram obtidos do banco de dados brasileiros da Dra. Délia Rodriguez Amaya, da Universidade Estadual de Campinas (Unicamp).

\section{Exame Clínico Oftalmológico}

Foi realizado exame clínico do globo ocular, sob luz ambiente, à procura de sinais de hipovitaminose A em todas as crianças. Numa subamostra (20\%), extraída aleatoriamente das crianças submetidas ao exame de sangue, foi realizado exame oftalmológico de biomicroscopia externa e de fundo de olho por especialistas da Unicamp (Dra. Alzira Delgado), para verificar sinais de xeroftalmia e conjuntivite.

\section{Avaliação Bioquímica}

\section{a. Coleta de Sangue}

Das amostras de sangue de 138 crianças, colhidas em jejum, em tubos Vacutainer sem anticoagulante, nos Centros de Saúde, foram separados os soros por centrifugação a $2500 \mathrm{rpm}$ por 15 minutos, três a quatro horas após a coleta. As amostras foram congeladas em gelo seco por 11 meses.

\section{b. Determinação dos Níveis Séricos de Retinol} O método utilizado para a extração do retinol foi o de Bieri et al. (1979), com algumas adaptações, seguido de determinação quantitativa por cromatografia líquida de alta eficiência (Clae), conforme recomendações feitas por Bieri et al. (1979) e Arroyave et al., (1982). Foi utilizado equipamento marca LDC/Milton Roy Company, com injetor tipo Rheodyne (alça de amostragem de $10 \mathrm{ml}$ ). O detector foi o Espectromonitor II mode- 
lo 1202 , operando na região ultravioleta a $325 \mathrm{~nm}$, e acoplado a um registrador modelo 3401/3402 (sensibilidade de 0.02 AUFS-Absorbance Units Full Scale). A separação cromatográfica foi feita em coluna de $150 \times 4.6 \mathrm{~mm} \mathrm{C}_{18}(5 \mu \mathrm{m})$, marca Lichrospher 100 (Merck), protegida por pré-coluna $\mathrm{C}_{18}(10 \mu \mathrm{m})$, Merck. Operou-se sempre de modo isocrático utilizando-se metanol:água (95:5) como fase móvel, filtrada e desgaseificada em banho ultrassônico. O fluxo da fase móvel foi de $1.0 \mathrm{ml} /$ min., gerando pressão de 1230 a 1500psi.

A classificação dos níveis sangüíneos de vitamina A foi feita com base no critério recomendado pelo Interdepartmental Committee on Nutrition for National Defense (ICNND, 1963).

c. Curva-Padrão de Retinol e Precisão Analítica

A curva-padrão foi preparada adicionando-se cinco diferentes concentrações de retinol $(0,52$; 1,$05 ; 1,57 ; 2,10 ; 2,62 \mu \mathrm{mol} / \mathrm{L}$ ) a um pool de soro. Logo depois, procedeu-se à extração do mesmo, em duplicata, com adição de quantidade constante do padrão interno $(1,4 \mu \mathrm{mol} / \mathrm{L})$. Todos os reagentes foram do grau Lichrosolv Merck. O padrão de retinol foi fornecido pela Roche Inc., e o de acetato de retinila adquirido da Sigma Chemical Co. A equação de regressão linear foi $\mathrm{y}=-0,0457+0,9619 x$.

A precisão do método para o mesmo dia foi determinada pela injeção de 10 extratos de um mesmo soro. $\mathrm{O}$ valor médio encontrado para o retinol foi $2,3 \mu \mathrm{mol} / \mathrm{L} \pm 0,14$, e o $\mathrm{CV}, 6,4 \%$. A precisão para diferentes dias, obtida pela extração em duplicatas de soro armazenado nas mesmas condições da amostra, foi $2,1 \mu \mathrm{mol} / \mathrm{L} \pm 0,20$, e o $\mathrm{CV}$, $8,7 \%$. A reprodutibilidade do aparelho foi verificada injetando-se durante 10 dias cada uma das cinco concentrações diferentes da solução-padrão contendo cerca de $1,4 \mu \mathrm{mol} / \mathrm{L}$ do padrão interno (acetato de retinila). O CV para o mesmo dia foi $4,1 \%$ e, para dias diferentes, $4,8 \%$.

A eficiência da extração média foi estimada em $89,8 \%$ (variação de 71 a 103\%).

\section{Processamento e Análise Estatística dos Dados}

As informações coletadas foram transferidas para um banco de dados Epi Info versão 5.01 b. Uma listagem contendo todos os dados do arquivo foi confrontada com os questionários para corrigir erros de digitação. Ao arquivo definitivo, aplicou-se um programa de detecção de erros, sendo o procedimento repetido exaustivamente.

No desenvolvimento das metodologias estatísticas utilizou-se o teste do Qui-Quadrado, e o teste $t$ de Student para comparação de médias de dois grupos no caso de variáveis quantitativas (Armitage \& Berry, 1987), aplicando-se o programa Statistical Package for Social Sciences (SPSS/PC+).

\section{RESULTADOS}

As Figuras 1 e 2 apresentam a distribuição dos indicadores antropométricos altura/idade $(\mathrm{n}=341)$ e peso/altura $(\mathrm{n}=320)$ das crianças examinadas.

FIGURA 1. Distribuição (\%) do índice Altura/Idade de 341 Crianças, de Ambos os Sexos, em Relação ao NCHS, Campinas, SP, 1991/1992

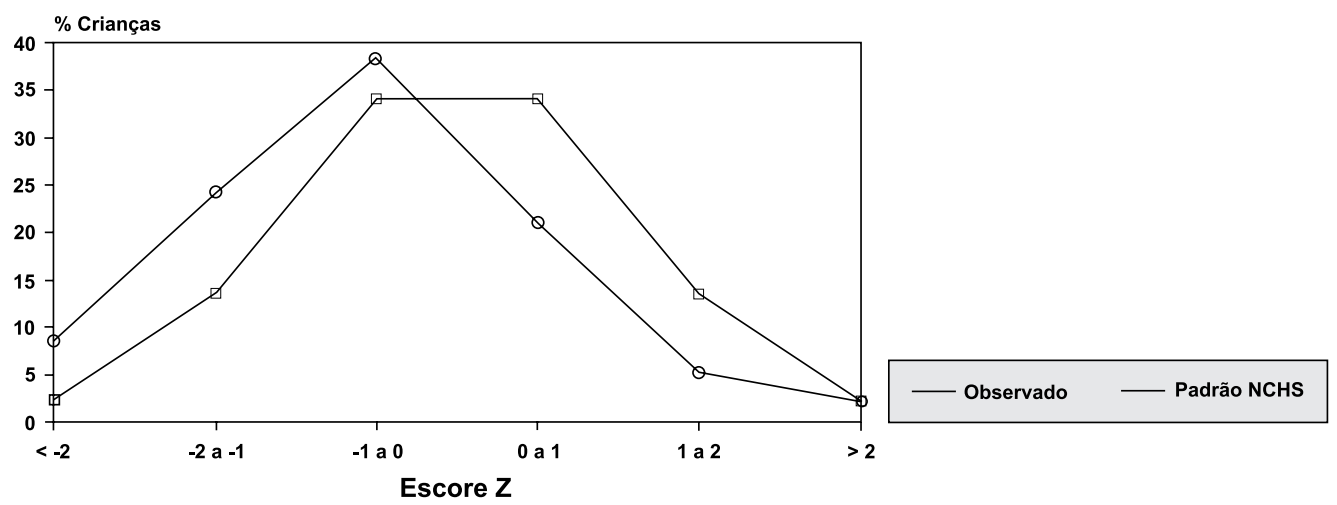


FIGURA 2. Distribuição (\%) do índice Peso/Altura de 320 Crianças, de Ambas os Sexos, em Relação ao NCHS, Campinas, SP, 1991/1992

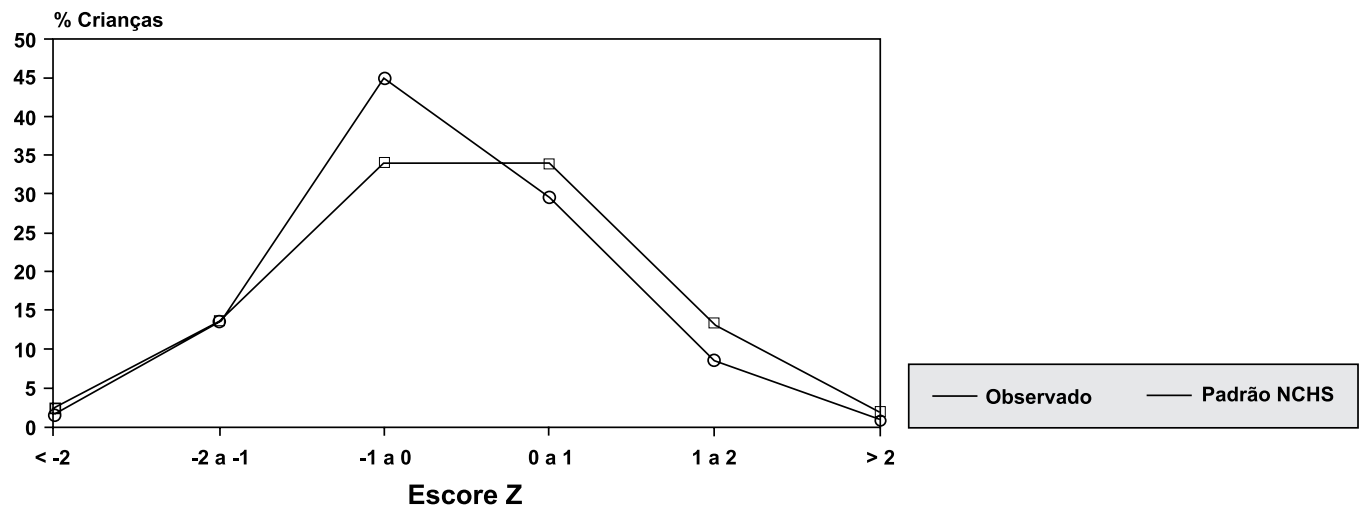

As Tabelas 1 e 2 mostram os resultados dos valores médios de retinol no soro, segundo a idade e o sexo das crianças, respectivamente.

Os resultados referentes à prevalência de hipovitaminose A, por faixa etária e sexo, en- contram-se nas Tabelas 3 e 4, respectivamente. $\mathrm{Na}$ Tabela 5 são apresentadas as estimativas dos Intervalos de Confiança para a prevalência de hipovitaminose A de acordo com o sexo e a faixa etária das crianças.

TABELA 1. Média, Desvio-Padrão, Valores Mínimos e Máximos dos Níveis de Retinol no Soro, segundo a Idade das Crianças. Campinas, SP, 1991/1992

\begin{tabular}{lcccc}
\hline \hline $\begin{array}{l}\text { Grupo Etário } \\
\text { (anos) }\end{array}$ & \multicolumn{4}{c}{ Retinol $(\mu \mathrm{moL})$} \\
\cline { 2 - 5 } & $\mathrm{N}$ o & $\mathrm{x} \pm \mathrm{DP}$ & Mínimo & Máximo \\
\hline 3 a 6 & 56 & $0,96 \pm 0,20$ & 0,48 & 1,64 \\
6 a 10 & 75 & $0,97 \pm 0,31$ & 0,48 & 1,68 \\
\hline \hline
\end{tabular}

$\mathrm{p}=0,980$ (Teste $t$ de Student)

TABELA 2. Média, Desvio-Padrão, Valores Mínimos e Máximos dos Níveis de Retinol no Soro, segundo o Sexo das Crianças, Campinas, Sao Paulo, 1991/1992

\begin{tabular}{lcccc}
\hline \hline & \multicolumn{4}{c}{ Retinol $(\mu \mathrm{moL})$} \\
\cline { 2 - 5 } Sexo & $\mathrm{N}^{0}$ & $\mathrm{x} \pm \mathrm{DP}$ & Mínimo & Máximo \\
\cline { 2 - 5 } Masculino & 57 & $0,92 \pm 0,28$ & 0,49 & 1,64 \\
Feminino & 74 & $1,00 \pm 0,31$ & 0,48 & 1,68 \\
\hline \hline
\end{tabular}

$\mathrm{p}=0,093$ (Teste $t$ de Student) 
TABELA 3. Porcentagem de Hipovitaminose A em Crianças de 3 a 10 Anos. Nível de Retinol no Soro, segundo a Idade, Campinas, São Paulo, 1991/1992

\begin{tabular}{lcccc}
\hline \hline & \multicolumn{4}{c}{ Retinol $(\mu \mathrm{moL})$} \\
\cline { 2 - 5 } $\begin{array}{l}\text { Grupo Etário } \\
\text { (anos) }\end{array}$ & \multicolumn{3}{c}{ Baixo } & \multicolumn{3}{c}{ Aceitável } \\
\cline { 2 - 5 } № & 9 & 6,9 & 47 & 35,9 \\
\hline a 6 & 14 & 10,7 & 61 & 46,6 \\
\hline a 10 & 23 & 17,6 & 108 & 82,4 \\
\hline \hline
\end{tabular}

$\mathrm{p}=0,87750$ (Teste Qui-quadrado)

TABELA 4. Porcentagem de Hipovitaminose A em Crianças de 3 a 10 Anos. Nível de Retinol no Soro, segundo o Sexo, Campinas, São Paulo, 1991/1992

\begin{tabular}{|c|c|c|c|c|}
\hline \multirow[b]{3}{*}{ Sexo } & \multicolumn{4}{|c|}{ Retinol ( $\mu \mathrm{moL})$} \\
\hline & \multicolumn{2}{|c|}{ Baixo } & \multicolumn{2}{|c|}{ Aceitável } \\
\hline & $\mathrm{N}^{\underline{\mathrm{Q}}}$ & $\%$ & $\mathrm{~N}^{\mathrm{o}}$ & $\%$ \\
\hline Masculino & 12 & 9,2 & 45 & 34,3 \\
\hline Feminino & 11 & 8,4 & 63 & 48,1 \\
\hline Total & 23 & 17,6 & 108 & 82,4 \\
\hline
\end{tabular}

$\mathrm{p}=0,48939$ (Teste Qui-Quadrado)

TABELA 5. Intervalo de Confiança $(n=131 ; \alpha=95 \%)$ para Prevalência de Hipovitaminose A, de Acordo com o Sexo e a Faixa Etária (anos), Campinas, São Paulo, 1991/1992

\begin{tabular}{ccc}
\hline \hline & Baixo & Aceitável \\
& $\rho \pm I C$ & $\rho \pm I C$ \\
\hline Sexo & $0,092 \pm 0,049$ & \\
$\bullet$ Masculino & $0,084 \pm 0,047$ & $0,343 \pm 0,087$ \\
$\bullet$ Feminino & & $0,630 \pm 0,083$ \\
\hline Faixa Etária & $0,069 \pm 0,043$ & \\
$3 \vdash 6$ & $0,107 \pm 0,053$ & $0,359 \pm 0,082$ \\
$6 \vdash 10$ & $0,176 \pm 0,065$ & $0,466 \pm 0,085$ \\
\hline Total & & $0,824 \pm 0,065$ \\
\hline \hline
\end{tabular}




\section{DISCUSSÃO}

\section{Aspectos Amostrais}

Das 350 crianças selecionadas, foram obtidas informações para 341. Das nove desclassificações, oito $(2,3 \%)$ o foram devido à recusa da família, e uma $(0,3 \%)$, à recusa da própria criança.

\section{Características da Amostra}

Sexo e Idade

Das 341 crianças estudadas, 175 (51,3\% eram do sexo feminino, e $166(48,7 \%)$ do sexo masculino. A média de idade das crianças, que variou de 3 a 10 anos, foi de $6,53 \pm 2,28$ anos.

\section{Renda per capita e Tamanho da Família}

As informações sobre a renda não foram fornecidas por $2,9 \%$ das famílias. Numa pesquisa sobre condições de saúde e nutrição das crianças de São Paulo, Monteiro (1988) refere ter encontrado $67,2 \%$ das crianças com renda familiar per capita inferior a um salário mínimo. Nesta pesquisa, encontraram-se poucas crianças $(12,1 \%)$ pertencentes a famílias com renda inferior a um salário mínimo per capita, uma maior proporção $(20,5 \%)$ entre um e dois salários mínimos per capita, e grande porcentagem $(49,3 \%)$ na faixa entre dois e cinco salários mínimos per capita. Embora as crianças possam parecer partícipes de uma situação privilegiada, as mesmas não apresentavam boas condições de vida. Em relação ao tamanho da família, foram registradas médias de $5,8 \pm 2,2$ pessoas por domícilio estudado, sendo $3,4 \pm 1,6$ a média do número de filhos por família.

\section{Instrução dos Pais}

Pelas informações sobre a instrução do pai e da mãe, verifica-se que a maioria das crianças $(84,5 \%)$ pertence a famílias cujo pai não teve nenhuma instrução ou cursou apenas os primeiros anos do ciclo primário. $\mathrm{O}$ primário completo foi registrado em apenas $4,1 \%$ dos pais. As informações foram obtidas para o pai da criança em cerca de 90,9\% dos casos; nos casos restantes, a família informou não ter convívio com o pai ou desconher a informação. Constatou-se também que quase $91 \%$ das crianças tinham mães sem nenhuma instrução ou com apenas os primeiros anos do curso primário. Por outro lado, o número de mães na categoria primário completo foi maior $(6,8 \%)$ do que o observado para os pais $(4,1 \%)$. Na categoria ginasial a instrução da mãe não diferiu da instrução do pai.

\section{Avaliação Antropométrica}

Os dados antropométricos (Figuras 1 e 2) foram analisados globalmente, de forma a evidenciar tendências da população-objeto em relação à curva de referencia do NCHS (OMS, 1983), recomendada para todos os países como referência e padrão. A rejeição espontânea registrada foi de $1 \%$. Na distribuição do índice altura para idade, nota-se um desvio da curva para a esquerda em relação à distribuição-referência, com cerca de $33,2 \%$ das crianças abaixo de -1 desvio padrão, demonstrando deficiências alimentares críticas na fase de crescimento.

Por outro lado, observando-se a distribuição das crianças quanto ao índice peso para altura, percebe-se leve desvio para a esquerda da referência, isto é, $15,3 \%$ da população encontrava-se abaixo da marca de -1 desvio padrão, o que sugere algum efeito de processo agudo de desnutrição na manutenção do peso. Martorell, citado por Horwitz (1989), sugere que o parâmetro peso-estatura é o melhor preditor de risco de saúde a curto prazo.

\section{Inquérito Alimentar (Recordatório de 24h)}

A avaliação nutricional da ingesta mostrou que o maior percentual de adequação foi para proteínas. Todas as faixas de idade apresentaram adequação superior a $100 \%$. Com relação à energia, a adequação média alcançou 87,76\%, sendo encontrados os maiores valores para o grupo etário de 3 a 6 anos $(93,91 \%)$, tornando-se progressivamente menor nos demais grupos (87,69\% para o grupo etário de 6 a 10 anos; $81,68 \%$ para o grupo etário de 10 a 11 anos). Os níveis do consumo de ferro e de vitamina A foram mui- 
to baixos, sendo especialmente reduzidos entre os 10 e 11 anos $(36,38 \%$ e $52,47 \%$, respectivamente). As adequações médias foram $66,13 \%$ para vitamina A e $42,14 \%$ para ferro. Esses resultados confirman o encontrado pelo IBGE (1982) e por Monteiro (1988). Moura (1984), avaliando a dieta de crianças de creches particulares de Campinas, São Paulo, também encontrou deficiência de ferro, vitamina A e energia, contra um excesso de proteínas.

\section{Avaliação do Estado Nutricional em Vitamina A}

\section{Exame Clínico Oftalmológico}

Nenhum sinal indicativo da presença de xeroftalmia foi encontrado na avaliação clínica ocular sob luz ambiente. Nas 26 crianças examinadas com aparelhos, as alterações observadas foram:

Hipermetropia

03 casos

Melanose da conjuntiva

02 casos

Crostas seborréicas

01 caso

Conjuntivite alérgica

01 caso

Astigmatismo

01 caso

Em 18 casos não houve alteração. Apesar de várias limitações, os métodos clínicos oculares são muito utilizados na avaliação do estado de vitamina A em crianças. Entretanto, essa metodologia é sensível apenas em casos de deficiência grave. Embora casos de xeroftalmia grave tenham sido relatados no Estado da Paraíba (Santos et al., 1983) e no Vale do Jequetinhonha, em Minas Gerais (Araújo et al., 1986), segundo Oliveira et al., (1990), os levantamentos sobre deficiência de vitamina A no Brasil, inferidos por meio de sinais clínicos, são inconsistentes.

\section{Prevalência de Hipovitaminose A pelas Dosagens de Retinol no Soro}

Do total da amostra $(\mathrm{n}=350)$, foram selecionadas 138 crianças para a coleta de sangue, sendo que, dessa, apenas 131 foram utilizadas para as dosagens de retinol. Nas restantes $(5,1 \%$ ou $\mathrm{n}=7$ ) houve hemólise.

O valor médio de retinol no soro das crianças estudadas foi $0,97 \pm 0,026 \mu \mathrm{mol} / \mathrm{L}$ ou $28 \mu \mathrm{g} / \mathrm{dL}$
(IC=0,92-1,02; 95\%), isto é, 66\% da média francesa para crianças normais (Malvy et al., 1989) e o dobro da média de crianças do Senegal (Amédée-Manesme \& De Maeyer, 1989). A mediana foi $0,93 \mu \mathrm{mol} / \mathrm{L}$ ou $27 \mu \mathrm{g} / \mathrm{dL}$.

Observando as faixas de variação, nota-se que os dados deste estudo mostram menor dispersão do que os da região de Tour, na França (Malvy et al., 1989) ou do Senegal (Amédée-Manesme $\&$ De Maeyer, 1989). Entre os fatores que podem determinar a amplitude de variação, devem ser considerados o tamanho da amostra, a abrangência geográfica, que por sua vez está relacionada com as fontes alimentares disponíveis, e o método de avaliação utilizado.

Flores et al. (1991), avaliando crianças de 2 a 6 anos de idade $(\mathrm{n}=544)$ de favelas do Recife, encontraram o valor médio de $0,82 \pm 0,24$ e mediana de $0,86 \mu \mathrm{mol} / \mathrm{L}$. A faixa de variação observada foi de 0,26 a $1,91 \mu \mathrm{mol} / \mathrm{L}$. A média está próxima da relatada neste estudo, para a faixa etária de 3 a 6 anos $(0,96 \pm 0,29 \mu \mathrm{mol} / \mathrm{L}$, Tabela 1$)$, embora a amplitude de variação tenha sido mais estreita para as crianças de Campinas (0,48 a 1,64 $\mu \mathrm{mol} / \mathrm{L}$ ou 14 a $47 \mu \mathrm{g} / \mathrm{dL})$. O fato de o menor valor ter sido mais alto e o maior, mais baixo do que os encontrádos por aqueles autores permite sugerir também que a amostra de Campinas seja mais homogênea.

Analisando os dados de acordo com a idade (Tabela 1), verificam-se valores médios de retinol praticamente semelhantes, não havendo diferenças estatisticamente significativas entre os pré-escolares e os escolares $(0,96$ e $0,97 \mu \mathrm{mol} / \mathrm{L}$, respectivamente). As diferenças observadas de acordo com o sexo (Tabela 2) apresentam a existência de valores ligeiramente mais elevados para as meninas $(1,01 \pm 0,31 \mu \mathrm{mol} / \mathrm{L})$ em relação aos meninos $(0,92 \pm 0,28 \mu \mathrm{mol} / \mathrm{L})$, mas sem diferenças significativas $(\mathrm{p}=0,093)$.

A influência do sexo nos níveis séricos de retinol é apontada por vários autores, havendo certa discordância. Alguns relatam maiores níveis de retinol em meninos (WHO, 1976), enquanto outros nas meninas (Suthuvoravoot \& Olson, 1974; Bloem et al., 1989).

Segundo Lewis et al. (1990) as diferenças observadas nos níveis sangüíneos de retinol entre os sexos são de menor magnitude em crianças quando comparadas com as relatadas em 
adultos. Valores de $0,07 \mu \mathrm{mol} / \mathrm{L}(2 \mu \mathrm{g} / \mathrm{dL})$ marcam a diferença máxima encontrada entre os meninos e meninas, enquanto diferenças de até $0,35 \mu \mathrm{mol} / \mathrm{L}$ já foram encontradas entre homens e mulheres.

Aceitando o critério proposto pelo ICNND (1963), que considera normais somente níveis acima de $20 \mu \mathrm{g} / \mathrm{dL}(>0,70 \mu \mathrm{mol} / \mathrm{L})$, sem distinção de sexo ou idade, constatou-se para o total da amostra prevalência de hipovitaminose A de $17,6 \%$ com níveis de retinol entre 0,35 e $0,70 \mu \mathrm{mol} / \mathrm{L}$ ( 10 e 20 $\mu \mathrm{g} / \mathrm{dl})$. Não foi detectada interação significativa entre o sexo e a idade das crianças com ou sem deficiência de vitamina A, embora maior prevalência tenha sido observada em crianças com idade igual ou superior a 6 anos (Tabelas 3 e 4).

Tem sido amplamente relatado que a prevalência de hipovitaminose A alcança índices alarmantes e que anualmente, os casos se repetem e até aumentam nos países em desenvolvimento. Mesmo nas suas formas leves ou moderadas, estima-se que a incidência se situe entre 6 e 7 milhões de casos, para prevalência de 20 a 40 milhões (Horwitz, 1989).

No Brasil, apesar de as informações sobre a extensão da hipovitaminose A serem insuficientes e geograficamente esparsas, Batista-Filho \& Cartagena (1985) consideram a deficiência de vitamina A um problema de saúde pública no país.

O ICNND (1965), que estudou o problema da hipovitaminose A no Brasil de forma mais abrangente, constatou freqüência global de $60 \%$ com níveis de retinol inferiores a $20 \mu \mathrm{g} / \mathrm{dL}$ e registrou ingesta alimentar capaz de fornecer apenas $50 \%$ das recomendações, em $42 \%$ das famílias. Estudo mais recente do IBGE (1982), por sua vez, verificou que as dietas dos brasileiros são geralmente indicativas de hipovitaminose. Santos et al. (1983) e Shrimpton (1989) vêm confirmando esses achados, embora não forneçam uma avaliação quantitativa da problemática como um todo.

No Estado de São Paulo, a prevalência de hipovitaminose A é apontada como sendo de proporções graves. Nos anos 80, Roncada et al. (1984) demonstraram que cerca de 30,2\% das crianças tinham níveis séricos de vitamina A abaixo de $20 \mu \mathrm{g} / \mathrm{dL}$.

Os achados bioquímicos relatados por Wilson et al. (1981) sobre a magnitude da prevalência da hipovitaminose A em crianças de 2 a 6 anos de idade revelaram quadro considerado mais grave: cerca de $73,9 \%$ dos examinados tinham níveis plasmáticos de vitamina $\mathrm{A}$ abaixo de $20 \mu \mathrm{g} / \mathrm{dL}$, enquanto $39,4 \%$, estavam com níveis abaixo de $10 \mu \mathrm{g} / \mathrm{dL}$.

Favaro et al. (1986) encontraram na região de Ribeirão Preto níveis circulantes de vitamina A semelhantes aos já relatados em crianças da Indonésia, para crianças consideradas de alto risco, ou seja, 47,2\% apresentaram níveis de retinol inferiores a $20 \mu \mathrm{g} / \mathrm{dL}$, e 1,8\%, níveis inferiores a $10 \mu \mathrm{g} / \mathrm{dL}$.

Na Guatemala, Gadomski et al. (1989), conduzindo trabalho semelhante ao nosso em grupo de pré-escolares, encontraram prevalência de $18 \%$ de níveis de retinol inferiores a $0,70 \mu \mathrm{mol} / \mathrm{L}$, sendo que nenhum caso com valores menores do que $0,35 \mu \mathrm{mol} / \mathrm{L}$ foi identificado. A prevalência da deficiência de vitamina A por meio de sinais clínicos é desconhecida, e não há relatos recentes de casos de cegueira nutricional na região. Apesar de a deficiência da vitamina na Guatemala ter apresentado índices menos severos do que os constantemente relatados na Indonésia e na Índia, a frequiência de níveis inferiores a $0,70 \mu \mathrm{mol} / \mathrm{L},(18 \%$ da população) é superior ao limite apontado pelo ICNND para um problema de saúde pública.

Lembramos ainda que, nos casos marginais o risco de evoluir para a categoria deficiente é muito grande, pois qualquer fator externo (queda súbita no consumo ou aumento nos requerimentos da vitamina) poderão diminuir acentuada e drasticamente os níveis de retinol sérico. Muitos trabalhos citam que os episódios infecciosos são capazes de promover rapidamente depleção das reservas hepáticas de vitamina A e queda nos níveis de retinol circulantes (Campos et al., 1987). Um fato interessante chamou a atenção de Campos et al. (1987): independente da presença de sinais clínicos, os níveis bioquímicos característicos de hipovitaminose A, em crianças pobres passaram de marginal (10-20 $\mu \mathrm{g} / \mathrm{dL})$ para deficiente $(<10 \mu \mathrm{g} / \mathrm{dL})$ após uma infecção.

Os resultados encontrados nesta pesquisa, a primeira dessa natureza na região de Campinas, mostram níveis séricos de retinol de $0,70 \mu \mathrm{mol} / \mathrm{L}$ em cerca de $17,6 \%$ da amostra, distribuídos em $6,9 \%$ para pré-escolares e $10,7 \%$ para escolares. Sugere-se, portanto, que a amostra da população infantil da periferia possui retinol sérico su- 
ficientemente baixo para ser considerada de alto risco, podendo evoluir facilmente para as formas mais graves da hipovitaminose A. Deve ser ressaltado, contudo, que nenhum caso de xeroftalmia ou mancha de Bitot foi detectado.

\section{RESUMO}

GONÇALVES-CARVALHO, C.M.R.; AMAYA-FARFAN, J.; WILKE, B. C. \& VENCOVSKY, R. Prevalência de

\section{Hipovitaminose A em Crianças da Periferia do} Município de Campinas, São Paulo, Brasil.

Cad. Saúde Públ., Rio de Janeiro, 11 (1): 85-96, jan/mar, 1995.

A prevalência de hipovitaminose A foi determinada pelos níveis séricos de retinol por cromatografia líquida de alta eficiência (Clae) em 131 crianças com idade entre 3 e 10 anos, residentes em 18 favelas do Município de Campinas, São Paulo, no período de abril de 1991 a fevereiro de 1992. A prevalência encontrada foi $17,6 \%$ com níveis entre 0,35 e $0,70 \mu \mathrm{mol} / \mathrm{L}$ (IC=11,1-24,1;95\%), o que indica a existência de certo risco de saúde pública. Entretanto, exames clínicos oftalmológicos não detectaram nenhum caso de xeroftalmia. Informações complementares sobre as características da amostra foram obtidas para 341 crianças. A renda per capita mostrou-se surpreendentemente alta. $\mathrm{O}$ consumo alimentar, segundo os critérios da FAO/WHO, só foi adequado para proteínas $(133,96 \%)$, sendo os menores valores de adequação aqueles encontrados para energia $(87,76 \%) \mathrm{e}$, principalmente, para vitamina A $(66,13 \%) \mathrm{e}$ ferro $(42,14 \%)$. Os indicadores altura/idade e peso/altura identificaram muitas crianças abaixo de -1 desvio padrão.

Palavras-Chave: Vitamina A; Hipovitaminose A; Retinol; Consumo Alimentar; Antropometria

\section{REFERÊNCIAS BIBLIOGRÁFICAS}

ANÇAO, M. S.; CUPPARI, L.; TUDISCO, E. S.; DRAIBE, S. A. \& SIGULEM, D., s/d. Sistema de Apoio a Decisão em Nutrição: Versão 1.0. São Paulo: Centro de Informática em Saúde da Escola Paulista de Medicina. (Mimeo.)

AMÉDÉE-MANESME, O. \& De MAEYER, E., 1989. Le Déficit en Vitamin A Strategies Diagnostiques et Thérapeutiques. Paris: Inserm-Orstom.

ARAÚJO, R. L.; ARAÚJO, M. B. D. G.; SIEIRO, R. O. ; MACHADO, R. D. P. \& LEITE, B. V., 1986. Diagnóstico de hipovitaminose A e anemia nutricional. Estudo realizado na população do Vale do Jequitinhonha, Minas Gerais. Revista Brasileira de Medicina, 43: 225-228.

ARMITAGE, P. \& BERRY, G., 1987. Statistical Methods in Medical Research. Oxford: Blackwell Scientific Publications.

ARROYAYE, G.; CHICHESTER, C. O.; FLORES, H.; GLOVER, J. G.; MEJIA, L. A.; OLSON, J. A.; SIMPSON, K. L \& UNDERWOOD, B., 1982. Biochemical Methodology for the Assessment of Vitamin A Status. Washington: IVACG.

BATISTA-FILHO, M. \& CARTAGENA, H. G., 1985. Epidemiologia das principais endemias carenciais no Brasil. In: Prioridades de Pesquisas Aplicadas ao Planejamento em Nutrição e Alimentação (CNPq/Seplan), pp. 87-132, Brasília, DF: CNPq/Seplan.

BIERI, J. G.; TOLLIVER, T. J. \& CATIGNANI, G. L., 1979. Simultaneous determination of alfatocopherol and retinol in plasma or red cells by high pressure liquid chromatography.American Journal of Clinical Nutrition, 32: 2143-2149.

BLOEM, M. W.; WEDEL, M.; EGGER, R. J.; SPEEK, A. J.; CHUSILP, K.; SAOWAKONTHA, S. \& SCHREURS, W. H. P., 1989. A prevalence study of vitamin A deficiency and xerophthalmia in Northeastern Thailand. American Journal of Epidemiology, 129: 1095-1103.

BLOEM, M. W.; WEDEL, M.; AGTMAAL, E. J. V.; SPEEK, A. J.; SAOWAKONTHA, S. \& SCHREURS, W. H. P., 1990. Vitamin A intervention: short-term effects of a single, oral, massive dose on iron metabolism. American Journal of Clinical Nutrition, 51: 76-79.

CAMPINAS (Prefeitura Municipal; Secretaria de Planejamento), 1993. População: Região de Campinas. Campinas: Prefeitura de Campinas. (Seminário de Dados, 1) (Mimeo.) 
CAMPOS, F. A. C. S.; FLORES, H. \& UNDERWOOD, B. A., 1987. Effect of an infection on vitamin A status of children as measured by the relative dose response (RDR). American Journal of Clinical Nutrition, 46: 91-94.

CARLIER, C.; MOULIAT-PELAT, J. P.; CECON, J. F.; MOUREY, M. S.; AMELINE, B.; FALL, M.; N'DIAYE, M. \& AMÉDÉE-MANESME, O., 1991. Prevalence of malnutrition and vitamin A deficiency in the Dioubel, Fatick, and Kaolak regions of Senegal: epidemiology study. American Journal of Clinical Nutrition, 53: 70-73.

COCHRAN, W. G., 1977. Samplig Techniques. $3^{\mathrm{a}}$ ed., New York: John Wiley.

FAO (Organização das Nações Unidas para a Agricultura e Alimentação), 1985. Necessidades de Energia y de Proteínas; Informe de una Reunião Consultiva Conjunta FAO/OMS/UNU de Expertos. Genebra: FAO. (Série Informes Técnicos, 724)

1988. Requirements of Vitamin A, Ion, Folate and Vitamin B12: Report of a Joint FAO/ WHO Expert Consultation. Genebra: FAO. (Food and Nutrition, 23) (Mimeo.)

FAVARO, R. M. D.; SOUZA, N. V.; BATISTAL, S. M.; FERRIANI, M. G. C.; DESAI, I. D. \& OLIVEIRA, J. E. D., 1986. Vitamin A status of young children in Southern Brazil. American Journal of Clinical Nutrition, 43: 852-858.

FLORES, H.; AZEVEDO, M. N. A.; CAMPOS, F. A. C. S.; BARRETO-LINS, M. C.; CAVALCANTI, A. A.; SALZANO, A. C.; VARELA, R. M. \& UNDERWOOD, B., 1991. Serum vitamin A distribuition curve for children aged 2-6 y known to have adequate vitamin A status: a reference population. American Journal of Clinical Nutrition, 54: 701-711.

GADOMSKI, A. M.; KJOLHEDE, C. L.; WITTPENN, J.; BULUX, J.; ROSAS, A. R. \& FORMAN, M. R., 1989. Conjunctival impression cytology (CIC) to detect subclinical vitamin A deficiency: comparison of CIC with biochemical assessments. American Journal of Clinical Nutrition, 49: 495-500.

HORWITZ, A., 1989. El costo de la malnutrición. In: Vigilancia Alimentaria y Nutricional en Ias Américas (Opas), pp. 19-54, Washington, DC: Opas. (Publicación Cientifica, 516)

IBGE (Fundação Instituto Brasileiro de Geografia e Estatística), 1982. Perfil Estatístico de Crianças e Mães no Brasil: Aspectos Nutricionais de 1974-1975. Rio de Janeiro: IBGE.

ICNND (Interdepartmental Committe on Nutrition for National Defense), 1963. Manual for Nutrition Surveys. 2a ed., Washington, DC: Government Printing Office.
1965. Northeast Brazil Nutrition Survey, March-May, 1963. Washington, DC: ICNND. (Mimeo.)

JORDAN, M. D., 1987. Anthropometric Software Package: Tutorial Guide and Handbook. Atlanta: Centers for Disease Control.

LEWIS, C. L.; MEDOWELL, M. A.; SEMPOS, C. T.; LEWIS, K. C. \& YETLEY, E. A., 1990. Relationship between age and serum vitamin A in children aged 4-1ly. American Journal of Clinical Nutrition, 51: 353-360.

MALVY, J. M. D.; MOUREY, M. S.; CARLIER, C.; CACES, P.; DOSTALOVA, L., MONTAGNON, B. \& AMÉDÉE-MANESME, O., 1989. Retinol, B-carotene and alfa-tocopherol status in a French population of health children. International Journal Vitamin of Nutrition Research, 59: 29-34.

MAZILLI, R. N. \& GANDRA, Y. R., 1981. Consumo alimentar de pré-escolares matriculados nos centros de educação e alimentação do pré-escolar (CEAPES) e de suas respectivas famílias. Revista de Saúde Pública, 15 (Supl.): 23-32.

MONTEIRO, C. A., 1988. Saúde e Nutrição das Crianças de São Paulo: Diagnóstico, Contrastes Sociais e Tendências. São Paulo: Hucitec/Edusp.

MOURA, E. C., 1984. Perfil nutricional de crianças de creches particulares do município de Campinas/ SP. Ciência/Assistência, 26: 39-42.

OLIVEIRA, J. E. D.; FAVARO, R. M. D. \& DESAI, I. D., 1990. Progress in the diagnosis of hypovitaminosis A: Clinical and biochemical correlations. Archivos Latinoamericanos de Nutrición, 11:333-348.

OMS (Organização Mundial da Saúde), 1983. Medicion del Cambio del Estado Nutricional. Genebra: OMS.

RONCADA, M. J.; WILSON, D.; OKANI, E. T. \& AMINO, S., 1984. Prevalência de hipovitaminose A em pré-escolares de município da área metropolitana de São Paulo, Brazil. Revista de Saúde Pública, 18: 218-224.

SANTOS, L. M. P.; DRICOT, J. M.: ASCIUTTI, L. S. \& DANS, D. C., 1983. Xerophthalmia in the state of Paraíba, Northeast of Brazil: Clinical findings. American Journal of Clinical Nutrition, 30: 139-144.

SHRIMPTON, R., 1984. Food consumption an dietary adequacy according to income in 1200 families, Manaus Amazonas, Brazil, 1973-1974. Archivos Latinoamericanos de Nutrición, 34: 615-629.

1989. Vitamin A deficiency in Brazil: perspectives for food production oriented interventions. Ecology Food Nutrition, 23: 261-271. 
SILVA, W., 1959. Inquérito sobre consumo de alimentos e nutrientes, avaliação do estado nutricional e situação econômica da população amazônica. Boletim do Comércio Nacional Alimentação, 42: 13.

SOLE, G.; BELAY, Y. \& ZEGEYE, B., 1987. Vitamin A deficiency in Southem Ethiopia. American Journal of Clinical Nutrition, 45: 780-784.

SOMMER, A., 1989a. Large dose vitamin A to control vitamin A deficiency. International Journal Vitamin of Nutrition Research, 30 (Suppl.): 37-41. 1989b. New imperatives for an old vitamin (A). Journal of Nutrition, 119: 96-100.

SOMMER, A.; HUSSAINI, G.; TARWOTJO, I. \& SUSANTO, D., 1983. Increased mortality in children with mild vitamin A deficiency. Lancet, 2: 585-588.

SOMMER, A.; TARWOTJO, I. \& KATZ, J., 1987. Increased risk of xerophthahnia following diarrhea and respiratory disease comparison to rat tissue retinol binding protein. American Journal of Clinical Nutrition, 45: 977-980.
SUTHUTVORAVOOT, S. \& OLSON, J. A., 1974. Plasma and liver concentrations of vitamin A in a normal population of urban Thaí. American Journal of Clinical Nutrition, 27: 883-889.

WILSON, D.; RONCADA, M. J.; NETTO LUI, A. \& NETTO, O. B., 1981. Hipovitaminose A em pré-escolares internados em uma instituição da capital do Estado de São Paulo, Brasil. Revista de Saúde Pública, 15: 395-400.

WILSON, D. \& RONCADA, M. J., 1985. Valor do corante vital rosa bengala como auxiliar no diagnóstico de xerose conjuntival em provas de triagem. Revista de Saúde Pública, 19: 321-335.

WHO (World Health Organization), 1976. Vitamin A deficiency and xerophthalmia. Geneva: WHO/ USAID. (Technical Report Series, 590) 